\title{
A note on intermittency for the fractional heat equation
}

\author{
Raluca M. Balan ${ }^{* \dagger} \quad$ Daniel Conus ${ }^{\ddagger}$
}

October 30, 2013

\begin{abstract}
The goal of the present note is to study intermittency properties for the solution to the fractional heat equation

$$
\frac{\partial u}{\partial t}(t, x)=-(-\Delta)^{\beta / 2} u(t, x)+u(t, x) \dot{W}(t, x), \quad t>0, x \in \mathbb{R}^{d}
$$

with initial condition bounded above and below, where $\beta \in(0,2]$ and the noise $W$ behaves in time like a fractional Brownian motion of index $H>1 / 2$, and has a spatial covariance given by the Riesz kernel of index $\alpha \in(0, d)$. As a by-product, we obtain that the necessary and sufficient condition for the existence of the solution is $\alpha<\beta$.
\end{abstract}

MSC 2010: Primary 60H15; secondary 37H15, 60H07

Keywords: fractional heat equation; fractional Brownian motion; Malliavin calculus; intermittency

\section{Introduction}

In this article we consider the fractional heat equation

$$
\left\{\begin{aligned}
\frac{\partial u}{\partial t}(t, x) & =-(-\Delta)^{\beta / 2} u(t, x)+u(t, x) \dot{W}(t, x), \quad t>0, x \in \mathbb{R}^{d} \\
u(0, x) & =u_{0}(x), \quad x \in \mathbb{R}^{d} .
\end{aligned}\right.
$$

where $\beta \in(0,2],(-\Delta)^{\beta / 2}$ denotes the fractional power of the Laplacian, and $u_{0}$ is a deterministic function such that

$$
a \leq u_{0}(x) \leq b \quad \text { for all } \quad x \in \mathbb{R}^{d}
$$

\footnotetext{
* Corresponding author. Department of Mathematics and Statistics, University of Ottawa, 585 King Edward Avenue, Ottawa, ON, K1N 6N5, Canada. E-mail address: rbalan@uottawa.ca

${ }^{\dagger}$ Research supported by a grant from the Natural Sciences and Engineering Research Council of Canada.

${ }^{\ddagger}$ Lehigh University, Department of Mathematics, 14 East Packer Avenue, Bethlehem, PA, 18109, USA. E-mail address: daniel.conus@lehigh.edu
} 
for some constants $b \geq a>0$. We let $W=\{W(\varphi) ; \varphi \in \mathcal{H}\}$ be a zero-mean Gaussian process with covariance

$$
E(W(\varphi) W(\psi))=\langle\varphi, \psi\rangle_{\mathcal{H}} .
$$

Here $\mathcal{H}$ is a Hilbert space defined as the completion of the space $C_{0}^{\infty}\left(\mathbb{R}_{+} \times \mathbb{R}^{d}\right)$ of infinitely differentiable functions with compact support on $\mathbb{R}_{+} \times \mathbb{R}^{d}$, with respect to the inner product $\langle\cdot, \cdot\rangle_{\mathcal{H}}$ defined by:

$$
\langle\varphi, \psi\rangle_{\mathcal{H}}=\alpha_{H} \int_{\left(\mathbb{R}_{+} \times \mathbb{R}^{d}\right)^{2}} \varphi(t, x) \psi(s, y)|t-s|^{2 H-2}|x-y|^{-\alpha} d t d x d s d y,
$$

where $\alpha_{H}=H(2 H-1), H \in(1 / 2,1)$ and $\alpha \in(0, d)$. We denote by $\dot{W}$ the formal derivative of $W$. The noise $W$ is spatially homogeneous with spatial covariance given by the Riesz kernel $f(x)=|x|^{-\alpha}$ and behaves in time like a fractional Brownian motion of index $H$. We refer to [2, 3, 5] for more details.

Let $G(t, x)$ be the fundamental solution of $\frac{\partial u}{\partial t}+(-\Delta)^{\beta / 2} u=0$ and

$$
w(t, x)=\int_{\mathbb{R}^{d}} u_{0}(y) G(t, x-y) d y
$$

be the solution of the equation $\frac{\partial u}{\partial t}+(-\Delta)^{\beta / 2} u=0$ with initial condition $u(0, x)=u_{0}(x)$. Note that

$$
G(t, \cdot) \text { is the density of } X_{t}
$$

where $X=\left(X_{t}\right)_{t \geq 0}$ is a symmetric Lévy process with values in $\mathbb{R}^{d}$. If $\beta=2$, then $X$ coincides with a Brownian motion $B=\left(B_{t}\right)_{t \geq 0}$ in $\mathbb{R}^{d}$ with variance 2 . If $\beta<2$, then $X$ is a $\beta$-stable Lévy process given by $X_{t}=B_{S_{t}}$, where $\left(S_{t}\right)_{t \geq 0}$ is a $(\beta / 2)$-stable subordinator with Lévy measure

$$
\nu(d x)=\frac{\beta / 2}{\Gamma(1-\beta / 2)} x^{-\beta / 2-1} 1_{\{x>0\}} d x .
$$

Due to (2) and (4), it follows that for all $t>0$ and $x \in \mathbb{R}^{d}$,

$$
a \leq w(t, x) \leq b .
$$

There is a rich literature dedicated to the case $H=1 / 2$, when the noise $W$ is white in time. We refer to 9,12 for some general properties, and to [11, 8, 7 ] for intermittency properties of the solution to the heat equation with this type of noise. Different methods have to be used for $H>1 / 2$, since in this case the noise is not a semi-martingale in time.

In the present article, we follow the approach of [13, 5] for defining the concept of solution. We say that a process $u=\left\{u(t, x) ; t \geq 0, x \in \mathbb{R}^{d}\right\}$ defined on a probability space $(\Omega, \mathcal{F}, P)$ is a mild solution of (1) if it is square-integrable, adapted with respect to the filtration induced by $W$, and satisfies:

$$
u(t, x)=w(t, x)+\int_{0}^{t} \int_{\mathbb{R}^{d}} G(t-s, x-y) u(s, y) W(\delta s, \delta y),
$$


where the stochastic integral is interpreted as the divergence operator of $W$ (see ([15]). Using Malliavin calculus techniques, it can be shown that the mild solution (if it exists) is unique and has the Wiener chaos decomposition:

$$
u(t, x)=\sum_{n \geq 0} I_{n}\left(f_{n}(\cdot, t, x)\right)
$$

where $I_{n}$ denotes the multiple Wiener integral (with respect to $W$ ) of order $n$, and the kernel $f_{n}(\cdot, t, x)$ is given by:

$$
\begin{aligned}
& f_{n}\left(t_{1}, x_{1}, \ldots, t_{n}, x_{n}, t, x\right)= \\
& \quad G\left(t-t_{n}, x-x_{n}\right) \ldots G\left(t_{2}-t_{1}, x_{2}-x_{1}\right) w\left(t_{1}, x_{1}\right) 1_{\left\{0<t_{1}<\ldots<t_{n}<t\right\}}
\end{aligned}
$$

(see page 303 of [13]). By convention, $f_{0}(t, x)=w(t, x)$ and $I_{0}$ is the identity map on $\mathbb{R}$.

The necessary and sufficient condition for the existence of the mild solution is that the series in (6) converges in $L^{2}(\Omega)$, i.e.

$$
S(t, x):=\sum_{n \geq 0} \frac{1}{n !} \alpha_{n}(t, x)<\infty,
$$

where

$$
\alpha_{n}(t, x)=n ! E\left|I_{n}\left(f_{n}(\cdot, t, x)\right)\right|^{2}=(n !)^{2}\left\|\tilde{f}_{n}(\cdot, t, x)\right\|_{\mathcal{H}^{\otimes n}}^{2}
$$

and $\tilde{f}_{n}(\cdot, t, x)$ is the symmetrization of $f_{n}(\cdot, t, x)$ in the $n$ variables $\left(t_{1}, x_{1}\right), \ldots$, $\left(t_{n}, x_{n}\right)$. If the solution $u$ exists, then $E|u(t, x)|^{2}=S(t, x)$. We refer to Section 4.1 of $\left[13\right.$ and Section 2 of $\left[5\right.$ ] for the details. Note that if $u_{0}(x)=u_{0}$ for all $x \in \mathbb{R}^{d}$, then the law of $u(t, x)$ does not depend on $x$, and hence $\alpha_{n}(t, x)=\alpha_{n}(t)$.

The goal of the present work is to give an upper bound for the $p$-th moment of the solution of (1) (for $p \geq 2$ ), and a lower bound for its second moment. In particular, this will show that, if $u_{0}(x)$ does not depend on $x$, then the solution $u$ of (1) is weakly $\rho$-intermittent, in a sense which has been recently introduced in [4], i.e. $\gamma_{\rho}(2)>0$ and $\gamma_{\rho}(p)<\infty$ for all $p \geq 2$, where

$$
\gamma_{\rho}(p)=\limsup _{t \rightarrow \infty} \frac{1}{t^{\rho}} \log E|u(t, x)|^{p}
$$

is a modified Lyapunov exponent (which does not depend on $x$ ), and

$$
\rho=\frac{2 H \beta-\alpha}{\beta-\alpha} .
$$

As a by-product, we obtain that the necessary and sufficient condition for the existence of the solution is $\alpha<\beta$. Note that this condition is equivalent to

$$
I_{\beta}(\mu):=\int_{\mathbb{R}^{d}}\left(\frac{1}{1+|\xi|^{2}}\right)^{\beta / 2} \mu(d \xi)<\infty
$$

with $\mu(d \xi)=c_{\alpha, d}|\xi|^{-d+\alpha} d \xi$, which is encountered in the study of equations with white noise in time. When $\beta=2$, (9) is called Dalang's condition (see [9]). 


\section{The result}

The goal of the present article is to prove the following result.

Theorem 2.1. The necessary and sufficient condition for equation (1) to have a mild solution is $\alpha<\beta$. If the solution $u=\left\{u(t, x) ; t \geq 0, x \in \mathbb{R}^{d}\right\}$ exists, then for any $p \geq 2$, for any $x \in \mathbb{R}^{d}$ and for any $t>0$ such that $p t^{2 H-\alpha / \beta}>t_{1}$

$$
E|u(t, x)|^{p} \leq b^{p} \exp \left(C_{1} p^{(2 \beta-\alpha) /(\beta-\alpha)} t^{\rho}\right)
$$

and for any $x \in \mathbb{R}^{d}$ and for any $t>t_{2}$,

$$
E|u(t, x)|^{2} \geq a^{2} \exp \left(C_{2} t^{\rho}\right),
$$

where $\rho$ is given by (8), $a, b$ are the constants given by (2), and $t_{1}, t_{2}, C_{1}, C_{2}$ are some positive constants depending on $d, \alpha, \beta$ and $H$.

Before giving the proof, we recall from [5] that

$$
\alpha_{n}(t, x)=\alpha_{H}^{n} \int_{[0, t]^{2 n}} \prod_{j=1}^{n}\left|t_{j}-s_{j}\right|^{2 H-2} \psi_{n}(\mathbf{t}, \mathbf{s}) d \mathbf{t} d \mathbf{s}
$$

where

$\psi_{n}(\mathbf{t}, \mathbf{s})=\int_{\mathbb{R}^{2 n d}} \prod_{j=1}^{n}\left|x_{j}-y_{j}\right|^{-\alpha} \tilde{f}_{n}\left(t_{1}, x_{1}, \ldots, t_{n}, x_{n}, t, x\right) \tilde{f}_{n}\left(s_{1}, y_{1}, \ldots, s_{n}, y_{n}, t, x\right) d \mathbf{x} d \mathbf{y}$

and we denote $\mathbf{t}=\left(t_{1}, \ldots, t_{n}\right), \mathbf{s}=\left(s_{1}, \ldots, s_{n}\right)$ with $t_{i}, s_{i} \in[0, t]$ and $\mathbf{x}=$ $\left(x_{1}, \ldots, x_{n}\right), \mathbf{y}=\left(y_{1}, \ldots, y_{n}\right)$ with $x_{i}, y_{i} \in \mathbb{R}^{d}$.

Note that the Fourier transform of $G(t, \cdot)$ is given by:

$$
\mathcal{F} G(t, \cdot)(\xi):=\int_{\mathbb{R}^{d}} e^{-i \xi \cdot x} G(t, x) d x=\exp \left(-t|\xi|^{\beta}\right), \quad \xi \in \mathbb{R}^{d}
$$

where $|\cdot|$ denotes the Euclidean norm in $\mathbb{R}^{d}$. Recall that for any $\varphi, \psi \in L^{1}\left(\mathbb{R}^{d}\right)$,

$$
\int_{\mathbb{R}^{d}} \int_{\mathbb{R}^{d}} \varphi(x) \psi(y)|x-y|^{-\alpha} d x d y=c_{\alpha, d} \int_{\mathbb{R}^{d}} \mathcal{F} \varphi(\xi) \overline{\mathcal{F} \psi(\xi)}|\xi|^{-d+\alpha} d \xi
$$

where $\mathcal{F} \varphi$ is the Fourier transform of $\varphi, c_{\alpha, d}=(2 \pi)^{-d} C_{\alpha, d}$ and $C_{\alpha, d}$ is the constant given by (21) (see Appendix A). This identity can be extended to functions $\varphi, \psi \in L^{1}\left(\mathbb{R}^{n d}\right)$ :

$$
\begin{aligned}
\int_{\mathbb{R}^{n d}} & \int_{\mathbb{R}^{n d}} \varphi(\mathbf{x}) \psi(\mathbf{y}) \prod_{j=1}^{n}\left|x_{j}-y_{j}\right|^{-\alpha} d \mathbf{x} d \mathbf{y}= \\
& c_{\alpha, d}^{n} \int_{\mathbb{R}^{n d}} \mathcal{F} \varphi\left(\xi_{1}, \ldots, \xi_{n}\right) \overline{\mathcal{F} \psi\left(\xi_{1}, \ldots, \xi_{n}\right)} \prod_{j=1}^{n}\left|\xi_{j}\right|^{-d+\alpha} d \xi_{1} \ldots \xi_{n} .
\end{aligned}
$$

We will use the following elementary inequality. 
Lemma 2.2. For any $t>0$ and $\eta \in \mathbb{R}^{d}$

$$
\int_{\mathbb{R}^{d}} e^{-t|\xi|^{\beta}}|\xi-\eta|^{-d+\alpha} d \xi \leq K_{d, \alpha, \beta} t^{-\alpha / \beta}
$$

where

$$
K_{d, \alpha, \beta}:=\sup _{\eta \in \mathbb{R}^{d}} \int_{\mathbb{R}^{d}} \frac{1}{1+|\xi-\eta|^{\beta}}|\xi|^{-d+\alpha} d \xi .
$$

Proof: Using the change of variable $z=t^{1 / \beta}(\eta-\xi)$, we have:

$$
\int_{\mathbb{R}^{d}} e^{-t|\xi|^{\beta}}|\xi-\eta|^{-d+\alpha} d \xi=t^{-\alpha / \beta} \int_{\mathbb{R}^{d}} e^{-\left|z-t^{1 / \beta} \eta\right|^{\beta}}|z|^{-d+\alpha} d z .
$$

The result follows using the inequality $e^{-x} \leq 1 /(1+x)$ for $x>0$.

Proof of Theorem 2.1; Step 1. (Sufficiency and upper bound for the second moment) Suppose that $\alpha<\beta$. We will prove that the series (7) converges, by providing upper bounds for $\psi_{n}(\mathbf{t}, \mathbf{s})$ and $\alpha_{n}(t, x)$.

By the Cauchy-Schwarz inequality, $\psi_{n}(\mathbf{t}, \mathbf{s}) \leq \psi_{n}(\mathbf{t}, \mathbf{t})^{1 / 2} \psi_{n}(\mathbf{s}, \mathbf{s})^{1 / 2}$. So it is enough to consider the case $\mathbf{t}=\mathbf{s}$. Let $u_{j}=t_{\rho(j+1)}-t_{\rho(j)}$ where $\rho$ is a permutation of $\{1, \ldots, n\}$ such that $t_{\rho(1)}<\ldots<t_{\rho(n)}$ and $t_{\rho(n+1)}=t$. Using (5), (11) and (13), and arguing as in the proof of Lemma 3.2 of [3], we obtain:

$$
\begin{gathered}
\psi_{n}(\mathbf{t}, \mathbf{t}) \leq b^{2} c_{\alpha, d}^{n} \int_{\mathbb{R}^{d}} d \eta_{1} \exp \left(-u_{1}\left|\eta_{1}\right|^{\beta}\right)\left|\eta_{1}\right|^{-d+\alpha} \int_{\mathbb{R}^{d}} d \eta_{2} \exp \left(-u_{2}\left|\eta_{2}\right|^{\beta}\right)\left|\eta_{2}-\eta_{1}\right|^{-d+\alpha} \\
\ldots \int_{\mathbb{R}^{d}} d \eta_{n} \exp \left(-u_{n}\left|\eta_{n}\right|^{\beta}\right)\left|\eta_{n}-\eta_{n-1}\right|^{-d+\alpha} .
\end{gathered}
$$

By Lemma 2.2, it follows that:

$$
\psi_{n}(\mathbf{t}, \mathbf{t}) \leq b^{2} c_{\alpha, d}^{n} K_{d, \alpha, \beta}^{n}\left(u_{1} \ldots u_{n}\right)^{-\alpha / \beta} .
$$

By inequality (26) (Appendix A), $K_{d, \alpha, \beta} \leq c_{\beta} I_{d, \alpha, \beta}$, where $c_{\beta}=2^{\beta / 2-1}$ and

$$
I_{d, \alpha, \beta}:=\int_{\mathbb{R}^{d}}\left(\frac{1}{1+|\xi|^{2}}\right)^{\beta / 2}|\xi|^{-d+\alpha} d \xi=\frac{(2 \pi)^{d} c_{d} \Gamma((\beta-\alpha) / 2) \Gamma(\alpha / 2)}{2 \Gamma(\beta / 2)}
$$

(see relation (24) and Remark A.3, Appendix A). Hence,

$$
\psi_{n}(\mathbf{t}, \mathbf{s}) \leq b^{2} C_{d, \alpha, \beta}^{n}[\beta(\mathbf{t}) \beta(\mathbf{s})]^{-\alpha /(2 \beta)}
$$

where $\beta(\mathbf{t})=u_{1} \ldots u_{n}, \beta(\mathbf{s})$ is defined similarly, and $C_{d, \alpha, \beta}>0$ is a constant depending on $d, \alpha, \beta$. Similarly to the proof of Proposition 3.5 of [5], we have:

$$
\alpha_{n}(t, x) \leq b^{2} C_{d, \alpha, \beta, H}^{n}(n !)^{\alpha / \beta} t^{n(2 H-\alpha / \beta)},
$$

where $C_{d, \alpha, \beta, H}>0$ is a constant depending on $d, \alpha, \beta, H$. Since $\alpha<\beta$, it follows that the series (7) converges and

$$
E|u(t, x)|^{2}=\sum_{n \geq 0} \frac{1}{n !} \alpha_{n}(t, x) \leq b^{2} \sum_{n \geq 0} \frac{C_{d, \alpha, \beta, H}^{n}}{(n !)^{1-\alpha / \beta}} t^{n(2 H-\alpha / \beta)} \leq b^{2} \exp \left(C_{0} t^{\rho}\right),
$$


for all $t>t_{0}$, where $C_{0}>0$ and $t_{0}>0$ are constants depending in $d, \alpha, \beta, H$. We used the fact that for any $a>0$ and $x>0$,

$$
\sum_{n \geq 0} \frac{x^{n}}{(n !)^{a}} \leq \exp \left(c_{0} x^{1 / a}\right) \quad \text { for all } \quad x>x_{0}
$$

where $x_{0}>0$ and $c_{0}>0$ are some constants depending on $a$.

Step 2. (Upper bound for the $p$-the moment) Note that $u(t, x)=\sum_{n \geq 0} J_{n}(t, x)$ in $L^{2}(\Omega)$, where $J_{n}(t, x)$ lies in the $n$-th order Wiener chaos $\mathcal{H}_{n}$ associated to the Gaussian process $W$ (see [15]). Hence,

$$
E|u(t, x)|^{2}=\sum_{n \geq 0} E\left|J_{n}(t, x)\right|^{2}=\sum_{n \geq 0} \frac{1}{n !} \alpha_{n}(t, x) .
$$

We denote by $\|\cdot\|_{p}$ the $L^{p}(\Omega)$-norm. We use the fact that for a fixed Wiener chaos $\mathcal{H}_{n}$, the $\|\cdot\|_{p}$ are equivalent, for all $p \geq 2$ (see the last line of page 62 of [15] with $q=p$ and $p=2$ ). Hence,

$$
\begin{aligned}
\left\|J_{n}(t, x)\right\|_{p} & \leq(p-1)^{n / 2}\left\|J_{n}(t, x)\right\|_{2}=(p-1)^{n / 2}\left(\frac{1}{n !} \alpha_{n}(t, x)\right)^{1 / 2} \\
& \leq b\left[(p-1) C_{d, \alpha, \beta, H}\right]^{n / 2} \frac{1}{(n !)^{(\beta-\alpha) /(2 \beta)}} t^{n(2 H \beta-\alpha) /(2 \beta)}
\end{aligned}
$$

using (14) for the last inequality. Using Minkowski's inequality for integrals (see Appendix A.1 of [16]) and inequality (15), we obtain that:

$$
\|u(t, x)\|_{p} \leq \sum_{n \geq 0}\left\|J_{n}(t, x)\right\|_{p} \leq b \exp \left(C_{1}(p-1)^{\beta /(\beta-\alpha)} t^{\rho}\right)
$$

if $p t^{2 H-\alpha / \beta}>t_{1}$, where the constants $C_{1}>0$ and $t_{1}>0$ depend on $d, \alpha, \beta, H$.

Step 3. (Necessity and lower bound for the second moment) Suppose that equation (11) has a mild solution $u$, i.e. the series (7) converges. In particular,

$$
\begin{aligned}
\infty>\alpha_{1}(t, x) & \geq a^{2} \alpha_{H} \int_{[0, t]^{2}} \int_{\mathbb{R}^{2 d}}|r-s|^{2 H-s}|y-z|^{-\alpha} G(s, y) G(r, z) d y d z d r d s \\
& =a^{2} \alpha_{H} c_{\alpha, d} \int_{\mathbb{R}^{d}}\left(\int_{0}^{t} \int_{0}^{t}|r-s|^{2 H-2} e^{-(r+s)|\xi|^{\beta}} d r d s\right)|\xi|^{-d+\alpha} d \xi \\
& \geq a^{2} \alpha_{H} c_{\alpha, d} c_{H} \int_{\mathbb{R}^{d}}\left(\frac{1}{1 / t+|\xi|^{\beta}}\right)^{2 H}|\xi|^{-d+\alpha} d \xi
\end{aligned}
$$

where we used (12) for the equality and Theorem 3.1 of [2] for the last inequality. From here, we infer that

$$
\alpha<2 H \beta \text {. }
$$

In particular, this implies that $\alpha<2 \beta$. 
Note that one can replace $\psi_{n}(\mathbf{t}, \mathbf{s})$ by $\psi_{n}(t \mathbf{e}-\mathbf{t}, t \mathbf{e}-\mathbf{s})$ in the definition (10) of $\alpha_{n}(t, x)$, where $\mathbf{e}=(1, \ldots, 1) \in \mathbb{R}^{n}$. By Lemma 2.2 of [1, we have:

$\psi_{n}(t \mathbf{e}-\mathbf{t}, t \mathbf{e}-\mathbf{s})=E\left[w\left(t-t^{*}, x+X_{t^{*}}^{1}\right) w\left(t-s^{*}, x+X_{s^{*}}^{2}\right) \prod_{j=1}^{n}\left|X_{t_{j}}^{1}-X_{s_{j}}^{2}\right|^{-\alpha}\right]$,

where $t^{*}=\max \left\{t_{1}, \ldots, t_{n}\right\}, s^{*}=\max \left\{s_{1}, \ldots, s_{n}\right\}$ and $X^{1}, X^{2}$ are two independent copies of the Lévy process $X=\left(X_{t}\right)_{t \geq 0}$ mentioned in the Introduction. (Lemma 2.2 of [1] was proved for $\beta=2$. The same proof is valid for $\beta<2$.)

Due to (5), it follows that

$$
a^{2} M_{n}(t) \leq \alpha_{n}(t, x) \leq b^{2} M_{n}(t)
$$

where

$$
M_{n}(t):=E\left[\alpha_{H}^{n} \int_{[0, t]^{2 n}} \prod_{j=1}^{n}\left|t_{j}-s_{j}\right|^{2 H-2} \prod_{j=1}^{n}\left|X_{t_{j}}^{1}-X_{s_{j}}^{2}\right|^{-\alpha} d \mathbf{t} d \mathbf{s}\right]=E\left(L(t)^{n}\right)
$$

and $L(t)$ is a random variable defined by:

$$
L(t):=\alpha_{H} \int_{0}^{t} \int_{0}^{t}|r-s|^{2 H-2}\left|X_{r}^{1}-X_{s}^{2}\right|^{-\alpha} d r d s .
$$

To prove that $L(t)$ is finite a.s., we show that its mean is finite. Note that $X_{r}^{1}-X_{s}^{2} \stackrel{d}{=} X_{r+s} \stackrel{d}{=}(r+s)^{1 / \beta} X_{1}$, and hence

$$
E[L(t)]=\alpha_{H} C_{d, \alpha, \beta} \int_{0}^{t} \int_{0}^{t}|r-s|^{2 H-2}(r+s)^{-\alpha / \beta} d r d s,
$$

where

$$
C_{d, \alpha, \beta}:=E\left|X_{1}\right|^{-\alpha}=\frac{c_{d} C_{\alpha, d}}{\beta} \Gamma(\alpha / \beta) .
$$

(The negative moment of the $\beta$-stable random variable $X_{1}$ can be computed similarly to (27), Appendix A.) Due to (16), it follows that $E[L(t)]<\infty$.

By (17), we have:

$$
a^{2} E\left(e^{L(t)}\right) \leq E|u(t, x)|^{2}=\sum_{n \geq 0} \frac{1}{n !} \alpha_{n}(t, x) \leq b^{2} E\left(e^{L(t)}\right) .
$$

We consider also the random variable

$$
\zeta(t):=\int_{0}^{t} \int_{0}^{t}\left|X_{r}^{1}-X_{s}^{2}\right|^{-\alpha} d r d s .
$$

Since $|r-s|^{2 H-2} \geq(2 t)^{2 H-2}$ for any $r, s \in[0, t], L(t) \geq \beta_{H} t^{2 H-2} \zeta(t)$, where $\beta_{H}=\alpha_{H} 2^{2 H-2}$. Hence $\zeta(t)$ is finite a.s. 
By the self-similarity (of index $1 / \beta$ ) of the processes $X^{1}$ and $X^{2}$, it follows that for any $t>0$ and $c>0$,

$$
\zeta(t) \stackrel{d}{=} c^{(2 \beta-\alpha) / \beta} \zeta(t / c) .
$$

In particular, for $c=t^{-(2 H-2) \beta /(2 \beta-\alpha)}$, we obtain that

$$
t^{2 H-2} \zeta(t) \stackrel{d}{=} \zeta\left(t^{\delta}\right), \quad \text { with } \quad \delta=\frac{2 H \beta-\alpha}{2 \beta-\alpha}
$$

and for $c=t$, we obtain that $\zeta(t) \stackrel{d}{=} t^{(2 \beta-\alpha) / \beta} \zeta(1)$. Hence,

$$
E\left(e^{L(t)}\right) \geq E\left(e^{\beta_{H} t^{2 H-2} \zeta(t)}\right)=E\left(e^{\beta_{H} \zeta\left(t^{\delta}\right)}\right) .
$$

The asymptotic behavior of the moments of $\zeta(t)$ was investigated in [6], under the condition $\alpha<2 \beta$. More precisely, under this condition, by relation (2.3) of [6], we know that:

$$
\lim _{n \rightarrow \infty} \frac{1}{n} \log \left\{\frac{1}{(n !)^{\alpha / \beta}} E\left[\zeta(1)^{n}\right]\right\}=\log \left(\frac{2 \beta}{2 \beta-\alpha}\right)^{(2 \beta-\alpha) / \beta}+\log \gamma,
$$

where $\gamma>0$ is a constant depending on $d, \alpha, \beta$. Hence, there exists some $n_{1} \geq 1$ such that for all $n \geq n_{1}, E\left[\zeta(1)^{n}\right] \geq c^{n}(n !)^{\alpha / \beta}$, where $c>0$ is a constant depending on $d, \alpha, \beta$. Consequently, for any $t>0$,

$$
E\left[\zeta(t)^{n}\right] \geq c^{n} t^{n(2 \beta-\alpha) / \beta}(n !)^{\alpha / \beta} \quad \text { for all } n \geq n_{1} .
$$

Hence, for any $\theta>0$,

$$
E\left(e^{\theta \zeta(t)}\right)=\sum_{n \geq 0} \frac{1}{n !} \theta^{n} E\left[\zeta(t)^{n}\right] \geq \sum_{n \geq n_{1}} \frac{1}{(n !)^{1-\alpha / \beta}} \theta^{n} c^{n} t^{n(2 \beta-\alpha) / \beta} .
$$

Using (18), (19) and (20), we obtain that:

$$
\infty>E|u(t, x)|^{2} \geq a^{2} E\left(e^{L(t)}\right) \geq a^{2} E\left(e^{\beta_{H} \zeta\left(t^{\delta}\right)}\right) \geq a^{2} \sum_{n \geq n_{1}} \frac{\beta_{H}^{n} c^{n} t^{n(2 H \beta-\alpha) / \beta}}{(n !)^{1-\alpha / \beta}} .
$$

This implies that $\alpha<\beta$. For any $x>0$ and $h \in(0,1)$, we note that

$$
E_{h}(x):=\sum_{n \geq 0} \frac{x^{n}}{(n !)^{h}} \geq\left(\sum_{n \geq 0} \frac{\left(x^{1 / h}\right)^{n}}{n !}\right)^{h}=\exp \left(h x^{1 / h}\right) .
$$

We denote $x_{t}=\theta c t^{(2 \beta-\alpha) / \beta}$ and $h=1-\alpha / \beta$. Writing the last sum in (20) as the sum for all terms $n \geq 0$, minus the sum $S_{t}$ with terms $n \leq n_{1}$, we see that for all $\theta>0$, and for all $t \geq t_{0}$,

$$
\begin{aligned}
E\left(e^{\theta \zeta(t)}\right) & \geq E_{h}\left(x_{t}\right)-S_{t} \geq \exp \left(h x_{t}^{1 / h}\right)-S_{t} \geq \frac{1}{2} \exp \left(h x_{t}^{1 / h}\right) \\
& \geq \exp \left(c_{0} \theta^{\beta /(\beta-\alpha)} t^{(2 \beta-\alpha) /(\beta-\alpha)}\right)
\end{aligned}
$$


where $c_{0}=h c^{1 / h}$ and $t_{0}>0$ is a constant depending on $\theta, \alpha, \beta$. Using this last inequality with $\theta=\beta_{H}$ and $t^{\delta}$ instead of $t$, we obtain that:

$$
E|u(t, x)|^{2} \geq a^{2} E\left(e^{\beta_{H} \zeta\left(t^{\delta}\right)}\right) \geq a^{2} \exp \left(C_{2} t^{\rho}\right),
$$

where $C_{2}=c_{0} \beta_{H}^{\beta /(\beta-\alpha)}$ depends on $d, \alpha, \beta, H$.

\section{A Some useful identities}

In this section, we give a result which was used in the proof of Theorem 2.1 for finding an upper bound for $\psi_{n}(\mathbf{t}, \mathbf{t})$. This result may be known, but we were not able to find a reference. We state it in a general context.

Following Definition 5.1 of [14, we say that a function $f: \mathbb{R}^{d} \rightarrow[0, \infty]$ is a kernel of positive type if it is locally integrable and its Fourier transform in $\mathcal{S}^{\prime}\left(\mathbb{R}^{d}\right)$ is a function $g$ which is non-negative almost everywhere. Here we denote by $\mathcal{S}^{\prime}\left(\mathbb{R}^{d}\right)$ the dual of the space $\mathcal{S}\left(\mathbb{R}^{d}\right)$ of rapidly decreasing, infinitely differentiable functions on $\mathbb{R}^{d}$.

The Riesz kernel defined by $f(x)=|x|^{-\alpha}$ for $x \in \mathbb{R}^{d} \backslash\{0\}$ and $f(0)=\infty$ (with $\alpha \in(0, d)$ ), is a kernel of positive type. Its Fourier transform in $\mathcal{S}^{\prime}\left(\mathbb{R}^{d}\right)$ is given by $g(\xi)=C_{\alpha, d}|\xi|^{-(d-\alpha)}$ where

$$
C_{\alpha, d}=\pi^{-d / 2} 2^{-\alpha} \frac{\Gamma((d-\alpha) / 2)}{\Gamma(\alpha / 2)}
$$

(see Lemma 1, page 117 of [16]).

Let $f$ be a continuous symmetric kernel of positive type such that $f(x)<\infty$ if and only if $x \neq 0$. By Lemma 5.6 of [14], for any Borel probability measures $\mu$ and $\nu$ on $\mathbb{R}^{d}$, we have:

$$
\int_{\mathbb{R}^{d}} \int_{\mathbb{R}^{d}} f(x-y) \mu(d x) \nu(d y)=\frac{1}{(2 \pi)^{d}} \int_{\mathbb{R}^{d}} \mathcal{F} \mu(\xi) \overline{\mathcal{F} \nu(\xi)} g(\xi) d \xi,
$$

where $\mathcal{F} \mu, \mathcal{F} \nu$ denote the Fourier transforms of $\mu, \nu$. In particular, if $\mu(d x)=$ $\varphi(x) d x$ and $\nu(d y)=\psi(y) d y$ for some density functions $\varphi, \psi$ in $\mathbb{R}^{d}$, then

$$
\int_{\mathbb{R}^{d}} \int_{\mathbb{R}^{d}} f(x-y) \varphi(x) \psi(y) d x d y=\frac{1}{(2 \pi)^{d}} \int_{\mathbb{R}^{d}} \mathcal{F} \varphi(\xi) \overline{\mathcal{F} \psi(\xi)} g(\xi) d \xi .
$$

This relation holds for arbitrary non-negative functions $\varphi, \psi \in L^{1}\left(\mathbb{R}^{d}\right)$. (To see this, we consider the normalized functions $\varphi /\|\varphi\|_{1}$ and $\psi /\|\psi\|_{1}$, where $\|\cdot\|_{1}$ denotes the $L^{1}\left(\mathbb{R}^{d}\right)$-norm.) Using the decomposition $\varphi=\varphi^{+}-\varphi^{-}$with nonnegative functions $\varphi^{+}, \varphi^{-}$, we see that (22) holds for any functions $\varphi, \psi \in$ $L^{1}\left(\mathbb{R}^{d}\right)$. In fact, (22) holds for any functions $\varphi, \psi \in L_{\mathbb{C}}^{1}\left(\mathbb{R}^{d}\right)$, replacing $\psi(y)$ by its conjugate $\overline{\psi(y)}$ on the left-hand side. (To see this, we write $\varphi=\varphi_{1}+i \varphi_{2}$ where $\varphi_{1}, \varphi_{2}$ are the real and imaginary parts of $\varphi$.) 
We consider the Bessel kernel (in $\mathbb{R}^{d}$ ) of order $\beta>0$ :

$$
G_{d, \beta}(x)=\frac{1}{\Gamma(\beta / 2)} \int_{0}^{\infty} u^{\beta / 2-1} e^{-u} \frac{1}{(4 \pi u)^{d / 2}} e^{-|x|^{2} /(4 u)} d u .
$$

Note that $G_{d, \beta}$ is a density function (see Remark A.3 below) and

$$
\mathcal{F} G_{d, \beta}(\xi)=\left(\frac{1}{1+|\xi|^{2}}\right)^{\beta / 2}, \quad \xi \in \mathbb{R}^{d}
$$

Moreover, $G_{d, \alpha} * G_{d, \beta}=G_{d, \alpha+\beta}$ for any $\alpha, \beta>0$ (see pages 130-135 of [16]).

The following result is an extension of relations (3.4) and (3.5) of [10] to the case of arbitrary $\beta>0$.

Lemma A.1. Let $f$ be a continuous symmetric kernel of positive type such that $f(x)<\infty$ if and only if $x \neq 0$. Let $\mu(d \xi)=(2 \pi)^{-d} g(\xi) d \xi$, where $g$ is the Fourier transform of $f$ in $\mathcal{S}^{\prime}\left(\mathbb{R}^{d}\right)$. Let $\beta>0$ be arbitrary. Then

$$
\begin{aligned}
& \int_{\mathbb{R}^{d}} G_{d, \beta}(x) f(x) d x=\int_{\mathbb{R}^{d}}\left(\frac{1}{1+|\xi|^{2}}\right)^{\beta / 2} \mu(d \xi):=I_{\beta}(\mu) . \\
& \text { If } I_{\beta}(\mu)<\infty \text {, then, for any } a \in \mathbb{R}^{d}, \\
& \int_{\mathbb{R}^{d}} e^{i a \cdot x} G_{d, \beta}(x) f(x) d x=\int_{\mathbb{R}^{d}}\left(\frac{1}{1+|\xi-a|^{2}}\right)^{\beta / 2} \mu(d \xi) .
\end{aligned}
$$

Proof: Relation (24) follows from (22) with $\varphi=\psi=G_{d, \beta / 2}$. On the left-hand side (LHS), we use the fact that $G_{d, \beta / 2} * G_{d, \beta / 2}=G_{d, \beta}$. On the right-hand side (RHS), we use (23) (with $\beta / 2$ instead of $\beta$ ).

To prove (25), we apply (22) to the complex-valued functions:

$$
\varphi(x)=\psi(x)=e^{i a \cdot x} G_{d, \beta / 2}(x) .
$$

The term on the LHS is

$$
\int_{\mathbb{R}^{d}} \int_{\mathbb{R}^{d}} e^{i a \cdot(x-y)} G_{d, \beta / 2}(x) G_{d, \beta / 2}(y) f(x-y) d x d y=\int_{\mathbb{R}^{d}} e^{i a \cdot x} f(x) G_{d, \beta}(x) d x,
$$

using Fubini's theorem. The application of Fubini's theorem is justified since

$$
\int_{\mathbb{R}^{d}} \int_{\mathbb{R}^{d}}\left|e^{i a \cdot(x-y)} G_{d, \beta / 2}(x) G_{d, \beta / 2}(y) f(x-y)\right| d x d y=\int_{\mathbb{R}^{d}} G_{d, \beta}(x) f(x) d x<\infty .
$$

For the term on the RHS, we use the fact that

$$
\mathcal{F} \varphi(\xi)=\int_{\mathbb{R}^{d}} e^{-i(\xi-a) \cdot x} G_{d, \beta / 2}(x) d x=\mathcal{F} G_{d, \beta / 2}(\xi-a)=\left(\frac{1}{1+|\xi-a|^{2}}\right)^{\beta / 4} .
$$


Corollary A.2. Let $(f, \mu)$ be as in Lemma A.1 and $\beta>0$ be arbitrary. Assume that $I_{\beta}(\mu)<\infty$. Then

$$
\sup _{a \in \mathbb{R}^{d}} \int_{\mathbb{R}^{d}}\left(\frac{1}{1+|\xi-a|^{2}}\right)^{\beta / 2} \mu(d \xi)=I_{\beta}(\mu)
$$

Consequently,

$$
\sup _{a \in \mathbb{R}^{d}} \int_{\mathbb{R}^{d}} \frac{1}{1+|\xi-a|^{\beta}} \mu(d \xi) \leq c_{\beta} I_{\beta}(\mu),
$$

where $c_{\beta}=2^{\beta / 2-1}$.

Proof: The fact that $I_{\beta}(\mu)$ is smaller than the supremum is obvious. To prove the other inequality, we take absolute values on both sides of (25) and we use the fact that $\left|\int \cdots\right| \leq \int|\cdots|$. For the last statement, we use the fact that $\left(1+|\xi-a|^{2}\right)^{\beta / 2} \leq c_{\beta}\left(1+|\xi-a|^{\beta}\right)$.

Remark A.3. The Bessel kernel $G_{d, \beta}(x)$ arises in statistics as the density of the random vector $X$ given by the following hierarchical model:

$$
X \mid U=u \sim N_{d}(0,2 u I) \quad U \sim \operatorname{Gamma}(\beta / 2,1)
$$

where $N_{d}(0,2 u I)$ denotes the $d$-dimensional normal distribution with covariance matrix $2 u I, I$ being the identity matrix. Hence, the term on the LHS of (24) is

$$
\int_{\mathbb{R}^{d}} G_{d, \beta}(x) f(x) d x=E[f(X)]=\frac{1}{\Gamma(\beta / 2)} \int_{0}^{\infty} u^{\beta / 2-1} e^{-u} E[f(X) \mid U=u] d u .
$$

This can be computed explicitly if $f(x)=|x|^{-\alpha}$ with $\alpha \in(0, d)$. First, note that if $Z \sim N_{d}(0,2 t I)$, then its negative moment of order $-\alpha$ is:

$$
E\left(|Z|^{-\alpha}\right)=\frac{1}{2} C_{\alpha, d} c_{d} \Gamma(\alpha / 2) t^{-\alpha / 2}
$$

where $c_{d}=2 \pi^{d / 2} / \Gamma(d / 2)$ is the surface area of the unit sphere in $\mathbb{R}^{d}$. To see this, we use the fact that $\mathcal{F} f(\xi)=C_{\alpha, d}|\xi|^{-d+\alpha} d \xi$ in $\mathcal{S}^{\prime}\left(\mathbb{R}^{d}\right)$. Hence,

$$
E\left(|Z|^{-\alpha}\right)=\int_{\mathbb{R}^{d}}|x|^{-\alpha} \frac{1}{(4 \pi t)^{d / 2}} e^{-|x|^{2} /(4 t)} d x=C_{\alpha, d} \int_{\mathbb{R}^{d}}|\xi|^{-d+\alpha} e^{-t|\xi|^{2}} d \xi
$$

and (27) follows by passing to the polar coordinates. We obtain that

$$
\int_{\mathbb{R}^{d}} G_{d, \beta}|x|^{-\alpha} d x=\frac{c_{\alpha, d} c_{d} \Gamma(\alpha / 2)}{2 \Gamma(\beta / 2)} \int_{0}^{\infty} u^{(\beta-\alpha) / 2-1} e^{-u} d u=\frac{C_{\alpha, d} c_{d} \Gamma((\beta-\alpha) / 2) \Gamma(\alpha / 2)}{2 \Gamma(\beta / 2)} .
$$

(Note that the integral is finite if and only if $\alpha<\beta$.) 


\section{References}

[1] Balan, R. M. (2009). A note on a Feynman-Kac type formula. Electr. Comm. Probab. 14, 252-260.

[2] Balan, R. M. (2012). Some linear SPDEs driven by a fractional noise with Hurst index greater than 1/2. Inf. Dimen. Anal. Quantum Probab. Rel. Fields 15.

[3] Balan, R. M. (2012). The stochastic wave equation with multiplicative fractional noise: a Malliavin calculus approach. Potential Anal. 36, 1-34.

[4] Balan, R. M. and Conus, D. (2013). Intermittency for the wave and heat equations driven by fractional noise in time. Preprint.

[5] Balan, R.M. and Tudor, C. A. (2010). The stochastic heat equation with fractional-colored multiplicative noise. J. Theor. Probab. 23, 834-870.

[6] Bass, R., Chen, X. and Rosen, M. (2009). Large deviations for Riesz potentials of additive processes. Ann. Inst. Henri Poincaré: Probab. ES Stat. 45, 626-666.

[7] Chen, L. and Dalang, R. C. (2013) Moments and growth indices for the nonlinear stochastic heat equation with rough initial conditions. (Preprint).

[8] Conus, D. and Khoshnevisan, D. (2012) On the existence and position of the farthest peaks of a family of stochastic heat and wave equations. Probab. Theory and Rel. Fields. 152, n3-4, 681-701.

[9] Dalang R.C. (1999) Extending martingale measure stochastic integral with applications to spatially homogeneous spde's. Electr. J. Probab. 4.

[10] Dalang, R. and Mueller, C. (2003). Some non-linear S.P.D.E.'s that are second order in time. Electr. J. Probab. 8, paper no. 1, 21 pages.

[11] Foondun, M. and Khoshnevisan, D. (2009) Intermittence and nonlinear parabolic stochastic partial differential equations. Electr. J. Probab. 14, paper no. $12,548-568$.

[12] Foondun, M. and Khoshnevisan, D. (2013). On the stochastic heat equation with spatially-colored random forcing. Trans. AMS 365, 409-458.

[13] Hu, Y. and Nualart, D. (2009) Stochastic heat equation driven by fractional noise and local time. Probab. Theory and Rel. Fields. 143, n1-2, 285-328.

[14] Khoshnevisan, D. and Xiao, Y. (2009). Harmonic analysis of additive Lévy processes. Probab. Th. Rel. Fields 145, 459-515.

[15] Nualart, D. (2006). Malliavin Calculus and Related Topics, Second Edition. Springer-Verlag, Berlin.

[16] Stein, E. M. (1970). Singular Integrals and Differentiability Properties of Functions. Princeton University Press. Princeton, New Jersey. 